\title{
Wave Number Selection for Incompressible Parallel Jet Flows \\ Periodic in Space
}

\author{
Jeffrey Hilton Miles* \\ National Aeronautics and Space Administration \\ Lewis Research Center \\ Cleveland, OH 44135
}

\begin{abstract}
The temporal instability of a spatially periodic, parallel flow of an incompressible inviscid fluid for various jet velocity profiles is studied numerically using Floquet Analysis. The transition matrix at the end of a period is evaluated by direct numerical integration. For verification, a method based on approximating a continuous function by a series of step functions was used. Unstable solutions were found only over a limited range of wave numbers and have a band type structure. The results obtained are analogous to the behavior observed in systems exhibiting complexity at the edge of order and chaos.
\end{abstract}

\section{Introduction}

The results of an investigation of the temporal instability of spatially periodic, parallel flow of an incompressible inviscid fluid for various velocity profiles are presented in this paper. This study is motivated by a desire to increase mixing in shear flows using multiple nozzles.

For many years the stability of jets, shear flows, and wakes have been extensively studied to understand the growth of a small, wave-like disturbance of the basic parallel inviscid flow $\mathbf{U}=U(y) \mathbf{i}$. For these unbounded flows, it is assumed that the flow is uniform as $y \rightarrow \pm \infty$.

The stability characteristics of spatially periodic, parallel flows of an incompressible fluid (both inviscid and viscous) were studied by Beaumont ${ }^{1}$. Beaumont considered periodic flows with $U(y)=U(y+\lambda)$ for some period $\lambda$ and all $y$. He considered broken line triangular and square velocity profiles and a continuous sinusoidal velocity profile. Miles ${ }^{2}$ extended this work in a study of the collective interaction of a compressible, periodic, parallel jet flow.

\footnotetext{
*Aerospace Engineer, Member AIAA

Copyright (C) 1997 by the American Institute of Aeronautics and Astronautics, Inc. No copyright is asserted in the United State under Title 17, U.S. Code. The U.S. Government has a royalty-free license to exercise all rights under the copyright claimed herein for Governmental Purposes. All other rights are reserved by the copyright owner.
}

In this paper, the stability characteristics of spatially periodic, parallel, inviscid jet flows of an incompressible fluid will be studied. A sketch of the nozzle geometry considered is shown in Fig. 1. A broken line V-shaped velocity profile, a continuous Gaussian velocity profile, and two continuous, roughly rectangular, velocity profiles, will be considered. For the steepest rectangular profile, stability will be studied for nozzle spacing-to-width ratios of $0.527,0.819,2.231$, and 7.183 .

An experimental study of an array of subsonic jets imbedded in a square network by Villermaux and Hopfinger $^{3}$ and Villermaux, Gagne, and Hopfinger ${ }^{4}$ found the jets exhibit a self-sustained oscillation. In addition, they found the oscillations synchronized by the fluctuating pressures gradient are strongly correlated over a short distance. Furthermore, measurements of the oscillations display the presence of large-wavelength travelling waves directed from the boundaries of the network to the center.

The study discussed herein may be considered to represent a one dimensional extrapolation to an array of rectangular jets separated by a distance $s$. The analysis depends on the jets being coherent spatially. In this linear temporal stability analysis of perturbations about the mean flow, it is the collective behavior of the periodic parallel jet flow that determines the behavior of the flow field.

The existence of unstable solutions for the broken line V-shaped velocity profile and the profiles studied by Beaumont ${ }^{1}$ suggested the possibility of unstable solutions for rectangular type profiles designed to have roughly the same scale. Beaumont showed that for the square broken line velocity profile all solutions are unstable. In this paper, we will show that for the $\mathrm{V}$-shaped velocity profile only non-growing neutral mode $\left(c_{i}=0\right)$ solutions exist. Stable solutions were found numerically for the continuous Gaussian velocity profile and two continuous, roughly rectangular, velocity profiles.

\section{Formulation of the problem}

The basic flow is assumed to be periodic with a maximum value $\widehat{U}_{2}$ and a minimum value $\widehat{U}_{1}$. The flow with velocity $\widehat{U}(\widehat{y})$ in the $\widehat{x}$ direction is assumed to be characterized by a length scale $\widehat{L}$ and a velocity scale 


$$
\Delta \widehat{U}=\widehat{U}_{2}-\widehat{U}_{1}
$$

The dimensionless variables used herein are $\dot{x}=\widehat{x} / \widehat{L}$, $y=\widehat{y} / \widehat{L}$, and $t=\widehat{t} \Delta \widehat{U} / \widehat{L}$.

Let $(\widehat{U}(y), 0)$ be the velocity of a steady, plane-parallel flow, where the $x$-axis is in the direction of the flow, and

$$
\widehat{U}(y)=\bar{U}+\frac{\Delta \widehat{U}}{2} f(y)
$$

where

$$
\bar{U}=\frac{\widehat{U_{2}}+\widehat{U_{1}}}{2}
$$

and $f$ is the velocity profile function which varies between \pm 1 .

The flow field is perturbed by introducing wave disturbances in the velocity and pressure with amplitudes that are a function of $y$. Thus

$$
(\tilde{u}, \tilde{v}, \tilde{p})=(u(y), v(y), p(y)) \exp [i(k x-\omega t)]
$$

where

$$
\begin{aligned}
k & =\widehat{k} \widehat{L}, \\
\omega & =\frac{\widehat{\omega} \widehat{L}}{\Delta \widehat{U}}, \\
\frac{\omega}{k} & =\frac{\widehat{\omega}}{\widehat{k} \Delta \widehat{U}}=\frac{\widehat{c}}{\Delta \widehat{U}}=\check{c}
\end{aligned}
$$

and we define $\check{c}$ as follows

$$
\check{c}=\frac{\widehat{c}}{\Delta \widehat{U}}=\frac{\vec{U}}{\Delta \widehat{U}}+\frac{c}{2} .
$$

By definition, $k$ is a real positive number that represents the wave number in the $x$-direction, $c$ is the disturbance velocity, $c=c_{r}+i c_{i}, c_{r}$ is the relative phase velocity, and $\omega_{i}=k c_{i} / 2$ is the amplification rate of the disturbance. From the equations of motion, if nonlinear and viscous terms are neglected, one can obtain the Rayleigh equation for the $y$-component of the perturbation velocity as follows:

$$
v^{\prime \prime}(y)-v(y)\left[\frac{f^{\prime \prime}(y)}{(f(y)-c)}+k^{2}\right]=0,
$$

where the primes denote differentiation with respect to $y$.

In this paper, $f(y)$ is periodic such that $f(y+\lambda)=f(y)$ where the period $\lambda$ used herein is the sum of a scaled nozzle width, $w_{N}=w_{N}^{*} / L^{*}$, and the scaled nozzle spacing, $s=s^{*} / L^{*}$. In this paper the period is scaled so that $\lambda=s+w_{N}=\left(s^{*}+w_{N}^{*}\right) / L^{*}=2 \pi$. The velocity profile $f(y)$ is not any exact solution of the Navier-Stokes equation, but it can be considered as a simple model of some real periodic flow.

The three continuous velocity profiles $f(y)$ studied herein are given by

$$
f_{i}(y)=1-2 g_{i}(\eta) \text {, for } i=3 \ldots 5
$$

where

$$
\eta=\Lambda\left(\frac{y}{\pi}-1\right)
$$

$\Lambda$ is the nozzle spacing parameter, $y$ goes from 0.0 to $2 \pi$,

$$
\begin{gathered}
g_{5}(\eta)=\frac{1}{\left[1+\left(\sinh \left(\frac{\eta}{\sinh (1)}\right)\right)^{18}\right]} \\
g_{4}(\eta)=\frac{1}{\left[1+(\eta)^{6}\right]}
\end{gathered}
$$

and

$$
g_{3}(\eta)=\exp \left(-(\eta)^{2}\right)
$$

The profile number system used $f_{1}=\sin (2 \pi y)$ and $f_{2}=$ $\cos (2 \pi y)$. The $f_{1}$ profile case was studied by Beaumont ${ }^{1}$.

The corresponding value of $d^{2} f_{5}(\eta) / d \eta^{2}$ is

$$
\begin{aligned}
d^{2} f_{5}(\eta) / d \eta^{2} & =\frac{-1296 \epsilon_{1}(\eta)^{34} \epsilon_{2}(\eta)^{2}}{\left[1+\epsilon_{1}(\eta)^{18}\right]^{3} \sinh (1)^{2}} \\
& +\frac{612 \epsilon_{1}(\eta)^{16} \epsilon_{2}(\eta)^{2}}{\left[1+\epsilon_{1}(\eta)^{18}\right]^{2} \sinh (1)^{2}} \\
& +\frac{36 \epsilon_{1}(\eta)^{18}}{\left[1+\epsilon_{1}(\eta)^{18}\right]^{2} \sinh (1)^{2}}
\end{aligned}
$$

where

$$
\begin{aligned}
\epsilon_{1}(\eta) & =\sinh \left(\frac{\eta}{\sinh (1)}\right) \\
\epsilon_{2}(\eta) & =\cosh \left(\frac{\eta}{\sinh (1)}\right)
\end{aligned}
$$

For $d^{2} f_{4}(\eta) / d \eta^{2}$ we have

$$
d^{2} f_{4}(\eta) / d \eta^{2}=\frac{-144 \eta^{10}}{\left[1+\eta^{6}\right]^{3}}+\frac{60 \eta^{4}}{\left[1+\eta^{6}\right]^{2}}
$$

For $d^{2} f_{3}(\eta) / d \eta^{2}$ we have

$$
\left.d^{2} f_{3}(\eta) / d \eta^{2}=4 \exp \left(-\eta^{2}\right)-8 \eta^{2} \exp \left(-\eta^{2}\right)\right) .
$$

Note that

$$
\frac{d^{2} f}{d y^{2}}=\left(\frac{\Lambda}{\pi}\right)^{2} \frac{d^{2} f}{d \eta^{2}}
$$

The form used for the $f_{5}$ function velocity profile was suggested by a velocity profile function used to study the instability of two-dimensional wakes by Monkewitz ${ }^{5}$. 
The manner in which altering the nozzle spacing parameter, $\Lambda$, changes the distance between nozzles will be discussed next. Let $\chi_{1}$ and $\chi_{2}$ be the roots of $f(y)$. The gap between the zero crossings is $s=\chi_{2}-\chi_{1}$. Changing $\Lambda$ shifts the roots of $f(y)$. The distance between the nozzle center lines is the period $\lambda=2 \pi$. The scaled jet width is $w_{N}=\lambda-s$. Results will be given in terms of the parameter $\Lambda$ and a parameter which is the ratio of the period, $\lambda=s+w_{N}$, to the scaled jet width, $w_{N}$ or the ratio of the gap width, $s$, to jet width, $w_{N}$. Note

$$
\frac{\lambda}{w_{N}}=\frac{s}{w_{N}}+1
$$

A value of 3 was used for $\Lambda$ for some of the results presented herein. For $\Lambda=3$, the corresponding velocity profiles and their first and second derivatives are shown in Fig. $2 a$ and $2 b$. Note that the gradients in these profiles are steep. For the steepest velocity profile, $f_{5}$, stability was studied using $\Lambda=3.0,2.3,1.5$, and1.18 which correspond to values of $\lambda / w_{N}=1.527,1.819,3.231$, and 8.183. Decreasing $\Lambda$ increases $\lambda / w_{N}$ and $s / w_{N}$.

\section{Floquet-Bloch theory}

Since the basic flow velocity profile, $f(y)$, is periodic, Eq. (1) is an example of a Floquet-Bloch problem. Differential equation, nonlinear system, and dynamical system textbooks frequently discuss the mathematics of solving Floquet-Bloch type problems ${ }^{6-16}$. The method has been applied in solid state physics ${ }^{17-21}$ to study the stability of a space vehicle ${ }^{22}$ and a helicopter rotor ${ }^{23-25}$. and to spatially periodic flow ${ }^{1,26-29}$. A survey of the spatially periodic flow literature is presented by $\mathrm{K}$. Gotoh and M.Y. Yamada ${ }^{30}$. The paper by Beaumont ${ }^{1}$ and the description of the Floquet-Bloch theorem by Hochstadt ${ }^{9}$ were particularly useful in guiding this research.

In this section, Eq. (1) is rewritten in a form used to obtain the numerical solutions discussed herein. The second order differential equation can be described by a system of first order differential equations. Let

$$
\begin{aligned}
v & =x_{1} \\
v^{\prime} & =x_{2}
\end{aligned}
$$

so that Eq. (1) can be rewritten as the system

$$
X^{\prime}=\mathbf{A} X
$$

where

$$
\mathbf{A}(\mathbf{y})=\left(\begin{array}{cc}
0 & 1 \\
D(y) & 0
\end{array}\right)
$$

$X$ is the vector $X=\left(x_{1}, x_{2}\right)^{T}$ and

$$
D(y)=\left[\frac{f(y)^{\prime \prime}}{(f(y)-c)}+k^{2}\right] .
$$

If $\mathbf{\Phi}(y)$ is a fundamental matrix solution of Eq. ( 7 ) such that

$$
\Phi(0)=\mathbf{I}
$$

where $\mathbf{I}$ is the identity matrix, then from the FloquetBloch theorem

$$
\boldsymbol{\Phi}(y+2 \pi)=\mu \boldsymbol{\Phi}(y) .
$$

For $y=0, \mu$ satisfies the matrix equation

$$
\boldsymbol{\Phi}(2 \pi)-\mu \mathbf{I}=0 .
$$

We now introduce two solutions of Eq.(1) with initial values at $y=0.0$

$$
\Phi(0)=\left[\begin{array}{cc}
\phi_{1}(0) & \phi_{2}(0) \\
\phi_{1}^{\prime}(0) & \phi_{2}^{\prime}(0)
\end{array}\right]=\left[\begin{array}{ll}
1 & 0 \\
0 & 1
\end{array}\right] .
$$

Next we seek the eigenvalues, $\mu$, of $\boldsymbol{\Phi}(2 \pi)$ from Eq. (8) as follows

$$
\begin{aligned}
& |\Phi(2 \pi)-\mu \mathbf{I}|=\left|\begin{array}{cc}
\phi_{1}(2 \pi)-\mu & \phi_{2}(2 \pi) \\
\phi_{1}{ }^{\prime}(2 \pi) & \phi_{2}{ }^{\prime}(2 \pi)-\mu
\end{array}\right| \\
= & \mu^{2}-\left(\phi_{1}(2 \pi)+\phi_{2}{ }^{\prime}(2 \pi)\right) \mu \\
& +\left(\phi_{1}(2 \pi) \phi_{2}{ }^{\prime}(2 \pi)-\phi_{2}(2 \pi) \phi_{1}{ }^{\prime}(2 \pi)\right) \\
= & \mu^{2}-\left(\phi_{1}(2 \pi)+\phi_{2}{ }^{\prime}(2 \pi)\right) \mu+1=0,
\end{aligned}
$$

where we have used the following relation

$\phi_{1}(2 \pi) \phi_{2}{ }^{\prime}(2 \pi)-\phi_{2}(2 \pi) \phi_{1}{ }^{\prime}(2 \pi)=|\mathbf{\Phi}(2 \pi)|=|\Phi(0)|=1$.

The independent solutions of Eq. (7) have the form

$$
\phi(y)=X(y) \exp \left(\frac{\log (\mu)}{2 \pi} y\right)=X(y) \exp (\Gamma y)
$$

The parameter $\Gamma$ specifies the period of the eigenfunction $\phi$. If $\Gamma$ is real the eigenfunction grows or decays at infinity. Consequently, only imaginary values of $\Gamma$ are acceptable. Thus, the eigenfunction oscillates in space and is called a continuous mode. The disturbance with $\Gamma_{i}=1 / n$, where $n$ is a nonzero integer, has a period $2 n \pi$. One with $\Gamma_{i}=0$ has the same period $2 \pi$ as the main flow, while an irrational value of $\Gamma_{i}$ means the disturbance is aperiodic. Note that the parameter $\Gamma$ does not appear in the flow equation, but is attributable to the Floquet-Bloch theorem.

Solutions of Eq. (7) are thus of the form

$$
\begin{aligned}
& X_{1}(y+2 \pi)=\mu_{1} X_{1}(y) \\
& X_{2}(y+2 \pi)=\mu_{2} X_{2}(y)
\end{aligned}
$$

where the eigenvalues $\mu_{1}$ and $\mu_{2}$ represent the zeros of Eq. (8), provided they are distinct.

In general, these solutions will not be periodic.

Conditions for periodic solutions can be found by letting $\mu_{1}=\exp \left(i \theta_{i}\right)$ and $\mu_{2}=\exp \left(-i \theta_{i}\right)$.

Then from equation (10) 


$$
\cos \left(\theta_{i}\right)=\delta / 2
$$

where

$$
\delta=\phi_{1}(2 \pi)+\phi_{2}{ }^{\prime}(2 \pi)
$$

Consequently, for a solution to be periodic, $\delta$ must be real and $|\delta|$ must be smaller than 2 so that $\theta_{i}$ is real.

The constants $\mu$ are termed the characteristic multipliers of the Floquet-Bloch system ( Eq.(1)) by Beaumont ${ }^{1}$ and the corresponding characteristic exponents are determined by the relation $\Gamma=\Gamma_{r}+i \Gamma_{i}=\frac{\log (\mu)}{2 \pi}=\frac{\theta_{r}}{2 \pi}+i \frac{\theta_{i}}{2 \pi}$ again in accordance with the definitions of Beaumont ${ }^{1}$.

Equation (1) can be put in a form that shows $k$ is an eigenvalue of a differential equation. ${ }^{31}$ From Eq. 1, we have

$$
v^{\prime \prime}-k^{2}\left[\frac{f^{\prime \prime}}{(f-c) k^{2}}+1\right] v=0
$$

where again the primes denote differentiation with respect to $y$.

Let $w=v$ and $z=v^{\prime} / k$ and $P(y, k)=-\left[\frac{f^{\prime \prime}}{(f-c) k^{2}}+1\right]$. Then

$$
\begin{aligned}
{\left[\begin{array}{l}
w^{\prime} \\
z^{\prime}
\end{array}\right] } & =k\left[\begin{array}{cc}
0 & 1 \\
-P & 0
\end{array}\right]\left[\begin{array}{l}
w \\
z
\end{array}\right] \\
& =k\left[\begin{array}{cc}
0 & 1 \\
-1 & 0
\end{array}\right]\left[\begin{array}{ll}
P & 0 \\
0 & 1
\end{array}\right]\left[\begin{array}{l}
w \\
z
\end{array}\right]
\end{aligned}
$$

or

$$
Z^{\prime}=k \mathbf{J H}(y, k) Z
$$

where $Z$ is the vector $Z=(w, z)^{T}$

$$
\mathbf{J}=\left[\begin{array}{cc}
0 & 1 \\
-1 & 0
\end{array}\right]
$$

and

$$
\mathbf{H}(y, k)=\left[\begin{array}{cc}
P(y, k) & 0 \\
0 & 1
\end{array}\right]
$$

Consequently, the wavenumber $\mathrm{k}$ can be viewed as an eigenvalue of the problem.

\section{Broken-line velocity profiles}

In this section, the stability of a broken-line V-shaped velocity profile and square-shaped velocity profile will be discussed. As shown by Beaumont ${ }^{1}$, one can use a procedure given by Rayleigh ${ }^{32}$ to obtain exact solutions for various periodic broken line velocity profiles. The method and the nature of the solutions is demonstrated using the Floquet-Bloch theory to obtain the simple explicit solutions for a broken line profiles. Beaumont ${ }^{1}$ discusses additional solutions for a broken line triangular profile and a broken line square velocity profile.
The Gaussian profile used herein has a resemblance to a V-shaped velocity profile. Consequently, in this section solutions for the V-shaped velocity profile are discussed. Since the approach is obscure more details on the solution are given in Appendix B.

The rectangular profile used herein has a resemblance to the square velocity profile discussed by Beaumont ${ }^{1}$. The square velocity case is treated anew herein since for the condition of continuity of normal velocity Beaumont ${ }^{1}$ uses continuity of $\phi /(U-c)$ rather than continuity of $\phi$. The condition that $\phi$ be continuous is presented in Appendix A where it is shown that the normal velocity, $v$, is $v=i k \phi$. Both approaches yield identical results for the triangular velocity profile case discussed by Beaumont and the V-profile treated herein which are continuous but have non continuous derivatives. However, the square velocity profile has discontinuities of the velocity profile and the stability solution presented herein is not the same as that derived by Beaumont ${ }^{1}$. Again, since the approach is obscure more details on the solution are given in Appendix C.

For this analysis the velocity potential formulation is used ${ }^{33}$ Thus, we have

$$
(U-c)\left(\phi^{\prime \prime}-k^{2} \phi\right)-U^{\prime \prime} \phi=0 \text {. }
$$

In Appendix A, the flow equations in terms of the velocity potential and velocity are shown to represent two different views of the same problem. The conditions of continuity of pressure and normal velocity indicate that at a discontinuity of $U$ or $U^{\prime}$

$$
\wp(\text { left })=\wp(\text { right })
$$

where

$$
\wp=(U-c) \phi^{\prime}-U^{\prime} \phi
$$

and

$$
\vee(l e f t)=\vee(\text { right })
$$

where

$$
\vee=\phi
$$

Note again that Beaumont ${ }^{1}$ expresses the condition of continuity of normal velocity using

$$
v=\frac{\phi}{(U-c)}
$$

The derivation of these equations is discussed in Appendix A.

\section{A. Broken-line V-shaped velocity profile}

The flow profile function $U(y)$ is given by 


$$
U(y)=\left\{\begin{array}{cc}
1-\frac{2}{\pi} y, & 0 \leq y \leq \pi \\
-3+\frac{2}{\pi} y, & \pi \leq y \leq 2 \pi
\end{array}\right.
$$

where $U(y+2 \pi)=U(y)$.

Using the two sets of initial conditions at $y=0$

$\left(\left(v, v^{\prime}\right)^{T}=(1,0)^{T}\right.$ and $\left.\left.\left(v, v^{\prime}\right)^{T}=(0,1)^{T}\right)\right)$ and using the matching equations at $y=\pi$, to solve the Rayleigh equation (Eq.(1)) at $y=2 \pi$ yields

$$
\begin{aligned}
\phi_{1}(2 \pi)= & \cosh (2 k \pi) \\
& -\frac{4}{k \pi(1+c)} \cosh (k \pi) \sinh (k \pi) \\
\phi_{2}{ }^{\prime}(2 \pi)= & \cosh (2 k \pi) \\
& -\frac{4}{k \pi(1+c)} \sinh (k \pi) \cosh (k \pi) .
\end{aligned}
$$

Consequently, using equation (12) and the angle sum relationship for $\sinh (\alpha+\beta)$

$$
\delta=2 \cosh (2 k \pi)-\frac{4}{k \pi(1+c)} \sinh (2 k \pi)
$$

From equation ( 11 ) $\delta$ is real. It then follows that since the value of $\delta_{i}$ is zero the value of $c_{i}$ is zero. Consequently, only neutrally stable solutions can exist. The value of $\delta$ for $c=0$ is shown in Fig. 3a. The value of $\delta$ is between \pm 2 only over a narrow range of $k$ values, $0.60949283 \leq$ $k \leq 0.6573595$.

For $c=0$, the value of $\delta$ vs $k$ and the stability curve. $\Gamma_{i}=\cos ^{-1}(\delta / 2) / 2 \pi$ vs $k$, based on equation 11 calculated from the following equation

$$
\begin{aligned}
\cos \left(\theta_{i}\right) & =\cos \left(2 \pi \Gamma_{i}\right)=\delta / 2 \\
& =\cosh (2 k \pi)-\frac{2}{k \pi} \sinh (2 k \pi)
\end{aligned}
$$

are shown in Fig. 3b and Fig. 3c.

Note again that a solution only exists between $k=$ 0.60949283 and $k=0.6573595$. Out side this narrow range of wave numbers the magnitude of $\delta$ is greater than 2 and no unstable solutions to the Floquet-Bloch problem exist.

\section{B. Broken-line square shaped velocity profile}

The flow $U(y)$ is given by:

$$
U(y)=\left\{\begin{array}{ccc}
1, & 0 \leq y \leq \frac{\pi}{2} \\
-1, & \frac{\pi}{2} \leq y \leq \frac{3 \pi}{2} \\
1, & \frac{3 \pi}{2} \leq y \leq 2 \pi
\end{array}\right.
$$

with $U(y+2 \pi)=U(y)$

Using the procedure discussed in Appendix C, the following equation for $\delta$ is derived:

$$
\delta=\frac{2\left[c^{2} \cosh (2 \pi k)-1\right]}{c^{2}-1}
$$

Then

$$
\begin{aligned}
\cos \left(\theta_{i}\right) & =\cos \left(2 \pi \Gamma_{i}\right) \\
& =\frac{\left[c^{2} \cosh (2 k \pi)-1\right]}{\left(c^{2}-1\right)}
\end{aligned}
$$

Note that solutions are available for only the neutral stability case where $c_{i}=0$ and $c=c_{r}$. For any $c_{i}$ not equal to zero $\cos \left(\theta_{i}\right)$ is complex and no unstable solutions exist.

The solution given by Beaumont ${ }^{1}$ is based on a different condition for velocity continuity and this solution shows that the whole of the $\left(k, \Gamma_{i}\right)$ plane is unstable.

In this section, analytic solutions were determined for two simple broken line velocity profiles. For these cases, only neutrally stable solutions were found. The numerical procedures used to obtain solutions for the continuous velocity profiles will be discussed next. The continuous velocity profiles are scaled to resemble the simple broken line velocity profile.

\section{Numerical calculations}

The specific point of interest is to determine for a given value of $c_{i}$ and $k$ if a value of $c_{\tau}$ exists such that the solution to Eq. (1) is unstable. In order to determine the unstable regions with a numerical implementation of Floquet-Bloch theory one must establish a grid in the $\left(c_{i}, k\right)$ parameter space and separately assess stability for each nodal point in the grid. For the results presented herein, the spacing in $k$ was 0.005 and the spacing in $c_{i}$ was 0.1 .

To investigate temporal stability for a given value of $c_{i}$ and $k$, iteration was used to vary the value of the phase velocity, $c_{r}$. At each iteration, the eigenvalues, $\mu$, of the matrix $\Phi(2 \pi)$ were then found and the corresponding characteristic multipliers $\Gamma$ calculated. The iteration was successful if $\delta_{i}=0$ so that $\Gamma_{r}=0$. Also, at each iteration a check was made that $\operatorname{det} \Phi(2 \pi)=1$.

The iteration procedure that selects appropriate values of $c_{r}$ consisted of the following steps. First, values of $c_{r}$ in the range between $-1<c_{r}<1$ at intervals of 0.02 are tried. Then these results are sorted to select the two solutions with the smallest value of $\left|\delta_{i}\right|$. The two corresponding values of $c_{r}$ are next used as guesses in a root finding IMSL subroutine (ZREAL) that tries to find two roots of $\left|\delta_{i}\left(c_{r}\right)\right|=0$. The solutions are then checked to see if $|\boldsymbol{\Phi}(2 \pi)|=1$ and $\delta_{r}$ is less than 2. Both solutions are retained if they exist. Note that while more than two solutions may exist, the procedure finds at most two solutions. This procedure is expensive and time consuming, but alternative procedures of comparable rigor, quality, and generality have not been forthcoming.

Two separate methods were used to evaluate the transition matrix at the end of a period $(y=2 \pi)$ so that $\delta$ and $|\Phi(2 \pi)|$ could be calculated. These will be discussed next. The numerical solutions were obtained using a CRAY YMP at the NASA Lewis Research Center. 


\section{A. Direct Numerical Integration}

In obtaining most of the results presented herein, equation (1) was integrated from $y=0$ to $y=2 \pi$ using a standard Runge-Kutta procedure (IMSL math library routine IVPRK). Typical numerical results obtained using numerical integration for velocity profiles $f_{3}, f_{4}$, and $f_{5}$ using $g_{3}, g_{4}$, and $g_{5}$ defined by equations 6,5 , and 4 are given in Tables 1 through 3. Each profile is calculated at a different pair of $k$ and $c_{i}$ values as noted in the Tables.

In order to confirm the proper functioning of the computer program and assess the accuracy of the results, typical cases were selected and verified using a spectral code and a method which approximated the periodic matrix $\mathbf{A}(y)$ by a series of step functions. In the next section, solutions obtained using the step function procedure will be compared to solutions obtained using direct numerical integration. Direct numerical integration proved faster than other integration procedures which were tried. In addition, it gives good results and copes with the steep gradients.

\section{B. Approximate Method of Determining $\Phi(2 \pi)$}

The approximate method to be discussed consists of approximating the periodic matrix $\mathbf{A}(y)$ by a series of step functions. To use the method, one evaluates the state transition matrix by dividing a period into many equal parts and treats the periodic matrix $\mathbf{A}(y)$ as a constant matrix in each part. Consequently, the differential equation is treated as a constant coefficient differential equation in each part. The method is described by $\mathrm{Hsu}^{34}$ and used by Friedmann and Silverthorn to study rotor blade flutter ${ }^{24}$ The period $2 \pi$ is divided into $\mathrm{K}$ intervals denoted by $y_{\kappa}, \kappa=0,1,2, \ldots K$. In the $\kappa$ th, interval the periodic coefficient matrix $\mathbf{A}(y)$ is replaced by a constant matrix $\mathbf{C}_{\kappa}=\mathbf{A}\left(y_{\kappa}+\frac{\Delta_{\kappa}}{2}\right)$ where $\Delta_{\kappa}=y_{\kappa}-y_{\kappa-1}$. Using the theory of differential equations with constant coefficients $^{35}$ the transition matrix of the system is given by

$$
\boldsymbol{\Phi}(2 \pi)=\prod_{\kappa=1}^{K} \exp \left(\Delta_{\kappa} \mathrm{C}_{\kappa}\right)
$$

The matrix exponential is defined as

$$
\exp \left(\Delta_{\kappa} \mathbf{C}_{\kappa}\right)=\mathbf{I}+\sum_{j=1}^{\infty} \frac{\left(\Delta_{\kappa} \mathbf{C}_{\kappa}\right)^{j}}{j !}
$$

For small spatial intervals this equation converges quickly, and the value of the matrix exponential can be approximated by a sum over a finite number of terms. However, for this problem, it can be shown that the matrix exponential has the exact solution ${ }^{35}$

$$
\exp \left(\Delta_{\kappa} \mathrm{C}_{\kappa}\right)=\left[\begin{array}{cc}
\cosh \left(\sqrt{D_{\kappa}} \Delta_{\kappa}\right) & \frac{\sinh \left(\sqrt{D_{\kappa}} \Delta_{\kappa}\right)}{\sqrt{D_{\kappa}}} \\
\sqrt{D_{\kappa}} \sinh \left(\sqrt{D_{\kappa}} \Delta_{\kappa}\right) & \cosh \left(\sqrt{D_{\kappa}} \Delta_{\kappa}\right)
\end{array}\right]
$$

The following results are presented to provide a more accurate view of the calculation errors than can be conveyed by the figures.

Exact matrix exponential step function approximation results as a function of the number of step function intervals for the three velocity profiles studied herein are presented in Tables 4, 5, and 6 for the same value of $k$ and $c_{i}$ used in corresponding Tables 1, 2, and 3. In addition, for velocity profile $f_{5}$, the computation time in seconds is shown. For the $f_{4}$ and $f_{3}$ velocity profiles results obtained using the exact second derivative are given in Tables $5 \mathrm{a}$ and $6 \mathrm{a}$ while results obtained using a numerically calculated second derivative are given in Tables 5b and $6 \mathrm{~b}$. For the step function approximation a range of intervals from $K=500$ to $K=40,000$ were used.

In Figures 4, 5, and 6 the discrepancy between the approximate step function result and the direct integration result are presented for $\delta, \Gamma$, and $c_{r}$ as a function of the number of intervals, $K$ for the three velocity profiles using the information presented in Tables 4 through 6 . The error scales with $10^{a} / K^{b}$ where $b$ is approximately unity. For each parameter $\delta, \Gamma$, and $c_{r}$, the value of $a$ increases as the velocity profile steepens. Figure 7 shows a plot of the calculation time as a function of the number of intervals, $K$. Note that the calculation time increases linearly with a slope equal approximately to 0.5 .

The integration results appear reasonable when compared to the step function approximation results. However, using the step function approximation takes large amounts of computer time to get results similar to that obtained using integration. Consequently, the direct numerical integration procedure was used for the calculations. Essentially, the method verified the direct numerical integration calculations.

\section{Results}

Typical stability curves based on the numerical computation results are shown in Figs. 8-13. Results are presented in pairs of plots for a range of $c_{i}$ values. The upper plot shows the phase speed as a function of growth rate, $\omega_{i}=k c_{i} / 2$. The lower plot shows the periodicity factor $\Gamma$ as a function of growth rate.

\section{A. Velocity profile effects}

In this section results obtained for the three velocity profiles obtained using $\Lambda=3$ are discussed. For each operating condition, the unstable wave is assumed to grow at the maximum rate possible. While the trace of solutions are shown for a range of $c_{i}^{\prime}$ values, emphasis is placed on the solution with the largest growth rate. For 
the $f_{3}$ profile (Fig. 8) solutions were found for $c_{i}$ in the range, $0.1 \leq c_{i} \leq 0.8$. For the $f_{4}$ profile (Fig. 9) solutions were found for $c_{i}$ in the range, $0.2 \leq c_{i} \leq 1$. Similarly, for the $f_{5}$ profile (Fig. 13) solutions were found for $c_{i}$ in the range, $0.1 \leq c_{i} \leq 0.92$.

For each profile the maximum growth rate occured at a different value of $c_{i}$. For the $f_{3}$ velocity, $\left(c_{i}\right)_{\max }=0.4$ (Fig. 8a), for the $f_{4}$ velocity, $\left(c_{i}\right)_{\max }=0.5$ (Fig. 9a), and for the $f_{5}$ velocity, $\left(c_{i}\right)_{\max }=0.9$ (Fig. 13a). The corresponding maximum growth rates are $\left(\omega_{i}\right)_{\text {max }}=$ $0.192,0.3038$, and 0.2453 . Thus while the range of $\left(c_{i}\right)_{\max }$ is large, the range of $\left(\omega_{i}\right)_{\max }$ is much smaller. The corresponding calculated relative phase speeds are $\left(c_{r}\right)_{\max }=0.1402,0.01143$, and 0.2111 . Thus the range of $\left(c_{r}\right)_{\max }$ is also small.

Examining plots of the characteristic exponent, $\Gamma_{i}$, verses the growth rate, $\omega_{i}$ (Figs $8 \mathrm{~b}, 9 \mathrm{~b}$, and $13 \mathrm{~b}$ ) shows most solutions are aperiodic. Hovever, for each profile solutions were found with $\Gamma_{i}$ near zero which have the same periodicity as the main flow. More importantly, the solution with the maximum growth rate has the same periodicity as the main flow.

The trace of points in Fig 9 for $c_{i}=0.6$ illustrates two interesting aspects of these results. First, note that the curve is double valued in the range $0 \leq \omega_{i} \leq 0.12$. Second, note that the trace consists of two islands of points one in the range $0 \leq \omega_{i} \leq 0.12$ and the other in the range $2.4 \leq \omega_{i} \leq 0.28$.

Another interesting feature is the occurence of instability waves with relative phase speeds near $c_{r}= \pm 0.5$. This feature can be seen in the trace of the $c_{i}=0.2$ curve in Fig 8 and the trace of the $c_{i}=0.6$ curve shown in Fig 9.

\section{B. Effects of spacing changes}

Figures 10-13 show results obtained using velocity profile $f_{5}$ with four different spacings $(\Lambda=1.18,1.5,2.3$, and 3 corresponding to the following ratios of nozzle spacing to nozzle width $s / \omega_{N}=7.183,2.231,0.81$ and 0.527 . Plots of the characteristic multipliers $\Gamma_{i}$ and the phase velocity $c_{r}$ as function of growth rate for a range of $c_{i}$ values are presented again as a function of growth rate. For these spacings, the maximum growth rate was at the following values of $\left(c_{i}\right)_{\max }=$ $0.3,0.7,0.9$, and 0.9 . The corresponding growth rates are $\left(\omega_{i}\right)_{\max }=0.1867,0.5547,0.2453$, and 0.2453 and the corresponding relative phase speeds are $\left(c_{r}\right)_{\max }=$ $0.5304,0.1913,0.06391$, and 0.2111 . The largest growth accurs for $\Lambda=1.5$ for this velocity profile.

The results given in Fig. 10 are unique. Results shown in Figs 11-13 were obtained for a large range of $c_{i}$ values at least from 0.2 to 0.9 . For $\Lambda=1.18$ the range of $c_{i}$ values was much smaller, $0.1 \leq c_{i} \leq 0.5$. Another difference is that for the largest growth rates, for the spacing $\Lambda=1.5,2.3$, and 3 , the relative phase velocity is in the range $0 \leq c_{r} \leq 0.3$. However, for the $\Lambda=1.18$ case for the largest growth rate $c_{r}$ is much larger $\left(c_{r}=0.5304\right)$. In addition, all solutions have positive $c_{r}$.

The traces given in Figs 11 through 13 are similar. They also traces which are broken up into islands as discussed earlier. The stability plot with $\Lambda=1.5$ is interesting in that the $\left(c_{i}\right)_{\max }=0.7$ trace represented by + is broken up into more than four islands.

Again in Figs 11 and 12 we note for a single trace at a particular value of $c_{i}$ the occurence of instability waves with relative phase speeds near $c_{r}= \pm 0.5$. These solutions switch between values creating islands of instability with a particular phase speed. This is shown in Fig. 11 for the trace $c_{r}=0.6$ and 0.7 and in Fig. 12 with the trace $c_{r}=0.6,0.7$ and 0.8 .

\section{Discussion}

In this section, some general remarks about the specific results will be made. Then, the nature of the solutions will be examined.

The collective behavior of spatially periodic, parallel, inviscid jet flows of an incompressible fluid supports unstable waves with many common temporal stability characteristics. The results indicate that for a wide variety of velocity profiles and nozzle spacings the unstable wave with maximum growth will have a relative phase velocity slightly greater than zero and have the same periodicity as the flow.

Extrapolation of these results to an experimental study of an array of subsonic jets imbedded in a square network by Villermaux and Hopfinger ${ }^{3}$ and Villermaux, Gagne, and Hopfinger ${ }^{4}$ suggest that the collective behavior observed might also be studied by Floquet-Bloch theory.

Systems of equations with coefficients periodic in time arise in the study of the stability of helicopter rotor blades flapping motions ${ }^{23-25}$, fluid column stability in the presence of periodic accelerations ${ }^{36}$, the instability of columns under periodic loading ${ }^{37}$, the stability of oscillatory Stokes layers ${ }^{38}$ and the instability of any system undergoing parametric excitation. Systems of equations periodic in space arise in solid state physics ${ }^{17-21}$, spatially periodic hydrodynamic flow ${ }^{1,26-29}$. The question of interest in many cases is

- determining the frequency of the instability which has the highest growth for systems undergoing periodic excitation

- the wavelength or the wave number of the instability which has the highest growth for systems with spatially periodic coefficients.

Theoretical models for systems where the variation is sinusoidal exhibit a continuous family of stable states. However, for the spatial variation considered herein one finds in addition islands of instability. The eigenfunctions of interest are the ones that do not vanish at infinity 
which are associated with the continuous eigenvalues and are called continuous modes. The new results obtained with steep velocity profiles indicate that these solutions can exist in discrete regions.

Beaumont ${ }^{1}$, showed that for sinusoidal velocity profiles the result is a region of instability and for a square velocity profile all points are unstable. However, it is shown herein that for velocity profiles that are almost square, Islands of instability are found. This simple formulation for almost square velocity profiles produces results that are surprising, complicated and essentially unpredictable.

These results can be categorized as follows. For the $v$, triangular, and sinusoidal velocity profiles, the $\left(c_{i}, k\right)$ space is divided into two regions one stable and one unstable and the space is highly ordered. For the square velocity profile all solutions are unstable and the space is highly disordered. In this paper, it is shown that by selecting certain velocity profiles, one observes a phase transition between highly ordered and completely disordered $\left(c_{i}, k\right)$ phase space, analogous to the phase transition between the solid and fluid states of matter. This analogy is also similar to the one made by Langton in the study of cellular automata ${ }^{39,40}$. Consequently, these results represent another example of a system exhibiting complexity at the edge of order and chaos ${ }^{40,41}$.

The procedure used to find solutions has some similarity to that used to study the Julia sets and the Mandelbrot sets which are fractal geometric objects. However, the search for solutions at each $\left(c_{i}, k\right)$ point is more difficult and the comprehensive method used requires more computer time. Consequently, enough work has not been done at this time to discern if the solutions are located in rigid domains or if the domain boundaries have a fractal nature. However, the basic procedure used involves finding the roots of $\left|\delta_{i}\left(c_{r}\right)\right|=0$. Newton's method provides a means to compute them using the following dynamical system

$$
\left\{\hat{C} ; f(z)=z-\frac{F(z)}{F^{\prime}(z)}\right\}
$$

Discussions of the application of Julia set theory to Newton's Method ${ }^{42}$ and a brief discussion by Shub on the theory of the complexity of equation-solving ${ }^{43}$ suggest the solutions might have a fractal nature. The sensitivity of the problem to spacing and the complexity of the results suggest that this is might be an interesting problem for someone working on the theory of the complexity of equation-solving.

\section{Conclusions}

For a range of amplification rates, solutions for the velocity profiles considered were found. For these cases, solutions exist over a limited range of wave numbers and have a band type structure.

These results represent another example of a system exhibiting complexity at the edge of order and chaos

\section{Appendix A: Formulation of Flow Equations}

The analytical study of the broken line V-shaped velocity profile uses a velocity potential formulation of Rayleigh's stability equation. The numerical study uses a velocity perturbation formulation of Rayleigh's stability equation. In this appendix, it is shown how these two formulations are related. The derivations starts with the two dimensional equations presented by Drazin and Reid $^{33}$ (Eq. 21.11) written in terms of $u$ and $v$ rather than $u$ and $w$ since in this paper we consider $U(y)$ instead of $U(z)$.

Thus we have

$$
\begin{gathered}
i k(U-c) u+U^{\prime} v=-i k p \quad \mathrm{~A} .1 \\
i k(U-c) v=-D p \quad \mathrm{~A} .2 \\
i k u+D v=0 \quad \mathrm{~A} .3
\end{gathered}
$$

where $/$ and $D$ represent the $d / d y$ operator. Introducing a stream function $\psi$ such that the two components of the disturbance velocity are given by

$$
\begin{gathered}
u=\frac{\partial \psi}{\partial y} \quad \mathrm{A.4} \\
v=-\frac{\partial \psi}{\partial x} \quad \mathrm{A.5}
\end{gathered}
$$

Let

$$
\psi=\phi(y) \exp ^{i k(x-c t)} \quad \text { A.6 }
$$

Then

$$
u=\phi^{\prime} \quad \mathrm{A} .7
$$

and the normal velocity is given by

$$
v=-i k \phi \quad \mathrm{A} .8
$$

Consequently, equation A.3 is automatically satisfied.

Substituting Equations A.7 and A.8 into Equation A.1 and solving for $p$ yields

$$
p=U^{\prime} \phi-(U-c) \phi^{\prime} . \quad \text { A.9 }
$$

Substituting equation A.8 and equation A.9 into equation A.2 yields a velocity potential formulation of Rayleigh's stability equation

$$
\phi^{\prime \prime}-\phi\left[\frac{U^{\prime \prime}}{(U-c)}+k^{2}\right]=0
$$

The velocity formulation of Rayleigh's stability equation is obtained by first solving equation $\mathrm{A} .2$ for $v$ 


$$
v=-\frac{D p}{i k(U-c)}
$$

Then equation A.3 is solved for $u$

$$
i k u=-D v
$$

Next one substitutes equation A.3 into equation A.1

$$
-(U-c) D v+U^{\prime} v=-i k p
$$

Then one takes the derivative of equation A.13 with respect to $y$ which yields

$$
(U-c)\left(-D^{2} v\right)+U^{\prime \prime} v=-i k D p
$$

Last, one uses equation A.2 to substitute for $D p$ to obtain

$$
D^{2} v-v\left[\frac{U^{\prime \prime}}{(U-c)}+k^{2}\right]=0
$$

The conditions for the continuity of pressure and normal velocity at a discontinuity of $U$ or $U^{\prime}$ for the broken line profile given by equations 15 thru 18 are easily derived from equations A.8 and A.9.

\section{Appendix B: Stability analysis for the broken line $\mathrm{V}$-shaped velocity profile}

The analytical study of the broken line V-shaped profile uses a velocity potential formulation of Rayleigh's stability equation. For the broken line profile $U^{\prime \prime}$ is zero and the resulting differential equation

$$
\phi^{\prime \prime}-\phi\left[k^{2}\right]=0 . \quad \text { B.1 }
$$

has solutions which are linear combinations of $\sinh (k y)$ and $\cosh (k y)$.

We now introduce two solutions of Eq. (B.1) $\phi_{1}(y)$ and $\phi_{2}(y)$ with initial values at $y=0.0$ given by equation 9 . In the region $0<y<\pi$ we have solutions

$$
\phi_{1}(y)=\cosh (k y) \quad \text { B. } 3
$$

and

$$
\phi_{2}(y)=\frac{1}{k} \sinh (k y) . \quad \text { B.4 }
$$

In the region $\pi<y<2 \pi$ we have solutions

$$
\phi_{1}=\cosh (k y)+M_{1 c} \sinh (k(y-\pi))
$$

and

$$
\phi_{2}=\frac{1}{k} \sinh (k y)+M_{2 s} \sinh (k(y-\pi)) \quad \text { B.6 }
$$

Next the conditions for continuity of pressure and normal velocity given by equations 15 thru 18 are applied.
Using the flow profile function given by equation 20 we have

$$
\begin{array}{rlr}
U\left(\pi_{-}\right) & =-1 & \text { B. } 7 \\
U^{\prime}\left(\pi_{-}\right) & =-\frac{2}{\pi} & \text { B.8 }
\end{array}
$$

$$
\begin{aligned}
U\left(\pi_{+}\right) & =-1 & \text { B. } 9 \\
U^{\prime}\left(\pi_{+}\right) & =\frac{2}{\pi} & \text { B. } 10
\end{aligned}
$$

Evaluating equations B.3 thru B.6 at $\pi$ we have

$$
\begin{array}{lr}
\phi_{1-}=\cosh (k \pi) & \text { B.11 } \\
\phi_{1-}^{\prime}=k \sinh (k \pi) & \text { B.12 }
\end{array}
$$

$$
\begin{gathered}
\phi_{1+}=\cosh (k \pi) \quad \text { B.13 } \\
\phi_{1+}^{\prime}=k \sinh (k \pi)+M_{1 c} k \quad \text { B. } \\
\phi_{2-}=\frac{1}{k} \sinh (k \pi) \quad \text { B.15 } \\
\phi_{2-}^{\prime}=\cosh (k \pi) \quad \text { B.16 }
\end{gathered}
$$

$$
\begin{aligned}
& \phi_{2+}=\frac{1}{k} \sinh (k \pi) \quad \text { B.17 } \\
& \phi_{2+}^{\prime}=\cosh (k \pi)+M_{2 s} k \quad \text { B.18 }
\end{aligned}
$$

Equations for continuity of the normal velocity ( equations 17 and 18) are satisfied at the velocity gradient discontinuity position $\pi$.

Equations for continuity of pressure (equations 15 and 16 ) at the velocity gradient discontinuity position $\pi$ yield values for $M_{1 c}$ and $M_{2 s}$.

$$
M_{1 c}=-\frac{4}{\pi} \frac{\cosh (k \pi)}{(1+c)} \quad \text { B.19 }
$$

and

$$
M_{2 s}=-\frac{4}{\pi k^{2}} \frac{\sinh (k \pi)}{(1+c)}
$$

At $2 \pi$ we have

$$
\phi_{1+}(2 \pi)=\cosh (2 \pi k)+M_{1 c} \sinh (\pi k)
$$

and

$$
\phi_{2+}^{\prime}(2 \pi)=\cosh (2 \pi k)+M_{2 s} k \cosh (\pi k)
$$

Using equation 12 and the angle sum relationship for $\sinh (\alpha+\beta)$ we can obtain equation 22 . 


\section{Appendix C: Stability analysis for the broken line square velocity profile}

Again, as with the broken line $\mathrm{V}$-shaped profile, the analytical study of the broken line square velocity profile uses a velocity potential formulation of Rayleigh's stability equation. Additionally, again, the broken line profile $U^{\prime \prime}$ is zero and the resulting differential equation (B.1) has solutions which are linear combinations of $\sinh (k y)$ and $\cosh (k y)$.

As before two solutions of Eq. (B.1) $\phi_{1}(y)$ and $\phi_{2}(y)$ are introduced with initial values at $y=0.0$ given by equation 9 .

In region one $0<y<\frac{\pi}{2}$ we have solutions

$$
\phi_{1,1}(y)=\cosh (k y) \quad \text { C.1 }
$$

and

$$
\phi_{2,1}(y)=\frac{1}{k} \sinh (k y)
$$

In the region $\frac{\pi}{2}<y<\frac{3 \pi}{2}$ we have solutions

$$
\phi_{1,2}=N_{1 c} \cosh \left(k\left(y-\frac{\pi}{2}\right)+M_{1 c} \sinh \left(k\left(y-\frac{\pi}{2}\right)\right)\right.
$$

and

$\phi_{2,2}=\frac{1}{k} N_{1 s} \sinh \left(k\left(y-\frac{\pi}{2}\right)\right)+M_{1 s} \cosh \left(k\left(y-\frac{\pi}{2}\right)\right)$

In the region $\frac{3 \pi}{2}<y<2 \pi$ we have solutions

$\phi_{1,3}=N_{2 c} \cosh \left(k\left(y-\frac{3 \pi}{2}\right)+M_{2 c} \sinh \left(k\left(y-\frac{3 \pi}{2}\right)\right)\right.$

and

$\phi_{2,3}=\frac{1}{k} N_{2 s} \sinh \left(k\left(y-\frac{3 \pi}{2}\right)\right)+M_{2 s} \cosh \left(k\left(y-\frac{3 \pi}{2}\right)\right)$

Next the conditions for continuity of pressure and normal velocity given by equations 15 thru 18 are applied. Note that $U^{\prime}=0$.

Using the flow profile function given by equation 20 we have

$$
\begin{array}{ll}
U\left(\frac{\pi}{2}\right)=1 & \text { C. } 7 \\
U^{\prime}\left(\frac{\pi}{2}\right)=0 & \text { C. } 8 \\
U\left(\frac{\pi}{2}_{+}\right)=-1 & \text { C. } 9 \\
U^{\prime}\left(\frac{\pi}{2}_{+}\right)=0 & \text { C. } 10
\end{array}
$$

Evaluating equations C. 1 thru C. 4 at $\frac{\pi}{2}$ we have

$$
\begin{aligned}
& \phi_{1,1-}=\cosh \left(k \frac{\pi}{2}\right) \\
& \phi_{1,1-}^{\prime}=k \sinh \left(k \frac{\pi}{2}\right)
\end{aligned}
$$

$$
\begin{array}{cc}
\phi_{1,2+}=N_{1 c} & \text { C.13 } \\
\phi_{1,2+}^{\prime}=k M_{1 c} & \text { C.14 } \\
\phi_{2,1-}=\frac{1}{k} \sinh \left(k \frac{\pi}{2}\right) & \text { C.15 } \\
\phi_{2,1-}^{\prime}=\cosh \left(k \frac{\pi}{2}\right) & \text { C.16 } \\
\phi_{2+}=M_{1 s} & \text { C.17 } \\
\phi_{2+}^{\prime}=N_{1 s} & \text { C.18 }
\end{array}
$$

Equations for continuity of the normal velocity ( equations 17 and 18) are satisfied at the velocity discontinuity position $\frac{\pi}{2}$ if

$$
N_{1 c}=\cosh \left(k \frac{\pi}{2}\right)
$$

$$
M_{1 s}=\frac{\sinh \left(k \frac{\pi}{2}\right)}{k}
$$

Equations for continuity of pressure (equations 15 and 16 ) at the velocity discontinuity position $\pi$ yield values for $M_{1 c}$ and $N_{1 s}$.

$$
M_{1 c}=\frac{c-1}{c+1} \sinh \left(k \frac{\pi}{2}\right)
$$

and

$$
N_{1 s}=\frac{c-1}{c+1} \cosh \left(k \frac{\pi}{2}\right) \quad \text { C. } 22
$$

Equations for continuity of the normal velocity (equations 17 and 18 ) and pressure (equations 15 and 16 ) yield at the velocity discontinuity position $\frac{3 \pi}{2}$ :

$$
\begin{aligned}
{\left[N_{1 c} \cosh (k \pi)\right.} & \left.+M_{1 c} \sinh (k \pi)\right] \\
-N_{2 c} & =0 \quad \mathrm{C} .23 \\
(-1-c)\left[N_{1 c} \sinh (k \pi)\right. & \left.+M_{1 c} \cosh (k \pi)\right] \\
-(1-c) M_{2 c} & =0 \quad \mathrm{C} .24 \\
& \\
{\left[\frac{N_{1 s}}{k} \sinh (k \pi)\right.} & \left.+M_{1 s} \cosh (k \pi)\right] \\
-M_{2 s} & =0 \quad \mathrm{C} .25 \\
(-1-c)\left[N_{1 s} \cosh (k \pi)\right. & \left.+M_{1 s} \sinh (k \pi)\right] \\
-(1-c) M_{2 s} & =0 \quad \mathrm{C} .26
\end{aligned}
$$

Solving for $N_{2 c}, M_{2 c}, M_{2 s}$, and $N_{2 s}$ and using the angle sum relationships for $\cosh (\alpha+\beta)$ and $\sinh (\alpha+\beta)$ we find:

$$
\begin{aligned}
& N_{2 c}=\frac{1}{1+c}\left[c \cosh \left(\frac{3 k \pi}{2}\right)+\cosh \left(\frac{k \pi}{2}\right)\right] \\
& M_{2 c}=\frac{1}{c-1}\left[c \sinh \left(\frac{3 k \pi}{2}\right)+\sinh \left(\frac{k \pi}{2}\right)\right] \\
& N_{2 s}=\frac{1}{c-1}\left[c \cosh \left(\frac{3 k \pi}{2}\right)-\cosh \left(\frac{k \pi}{2}\right)\right] \\
& M_{2 s}=\frac{1}{k(c+1)}\left[c \sinh \left(\frac{3 k \pi}{2}\right)-\sinh \left(\frac{k \pi}{2}\right)\right]
\end{aligned}
$$


At $2 \pi$ we have

$$
\phi_{1+}(2 \pi)=N_{2 c} \cosh \left(\frac{\pi k}{2}\right)+M_{2 c} \sinh \left(\frac{\pi k}{2}\right)
$$

and

$$
\phi_{2+}^{\prime}(2 \pi)=N_{2 s} \cosh \left(\frac{\pi k}{2}\right)+M_{2 s} k \sinh \left(\frac{\pi k}{2}\right) \quad \text { C.32 }
$$

Using equation 12 and the angle sum relationships we can obtain equation 25 .

\section{References}

${ }^{1}$ D. N. Beaumont. The stability of spatially periodic flows. J. Fluid Mech., 108 :461-474, 1981.

2 Jeffrey Hilton Miles. Collective Interaction of a Compressible Periodic Parallel Jef Flow. Technical Report AIAA$97=0150$, AIAA, 1997.

${ }^{3}$ E. Villermaux and E.J. Hopfinger. Periodically arranged co-flowing jets. J. Fluid Mech., 263 No.:63-92, 1994.

${ }^{4}$ E. Villermaux, Y. Gagne, and E.J. Hopfinger. Self sustained oscillations and collective behaviours in a lattice of jets. Applied Scientific Research, 51 :243-248, 1993.

5 Peter A. Monkewitz. The absolute and convective nature of instability in two-dimensional wakes at low reynolds numbers. Phys. Fluids, 31No.5:999-1006, May 1988.

${ }^{6} \mathrm{E}$. L. Ince. Ordinary differential equations. Dover, 1926,1956. pp. 381-382.

${ }^{7}$ Harold T. Davis. Introduction to nonlinear differential and integral equations. Dover, 1960. pp. 300-303.

8 Jack K. Hale. Oscillations in Nonlinear Systems. Dover Publications, Inc., 1963. 19,106-109.

${ }^{9}$ Harry Hochstadt. Differential Equations. Dover, 1963. pp. 195-200.

${ }^{10}$ Thomas L. Saaty and Joseph Bram. Nonlinear Mathematics. Dover Publications, Inc. New York, 1964,1981. pp. 219 235.

${ }^{11}$ Chihiro Hayashi. Nonlinear Oscillations in Physical Systems. McGraw-Hill Book Company, 1964. pp. 82-97.

12 John Guckenheimer and Philip Holmes. Nonlinear Oscillations, Dynamical Systems, and Bifurcations of Vector Fields. Springer-Verlag, 1983. pp. 24-25.

${ }^{13}$ M. G. Krein. Four papers on ordinary differential equations. American Mathematical Society translations; ser. 2, v. 120, 1983.

${ }^{14} \mathrm{~K}$. Arrowsmith and C. M. Place. An Introduction to Dynamical Systems. Cambridge University Press, 1990. pp. 89-93,114.

${ }^{15}$ Ferdinand Verhulst. Nonlinear Differential Equations and Dynamical Systems. Springer-Verlag, 1990. pp. 80-87,239240.

${ }^{16}$ Daniel Zwillinger. Handbook of Differential Equations. Academic Press, 1992. pp. 448-118.

${ }^{17}$ Mendel Sachs. Solid State Theory. Dover, 1963. pp. 189198.

${ }^{18}$ Charles Kittel. Introduction to SOLID STATE PHYSICS. John Wiley \& Sons, Inc., 1961. pp. 279-301.

${ }^{19}$ Gregory H. Wannier. Elements of Solid State Theory. Cambridge University Press, 1960. pp. 130-144.

${ }^{20} \mathrm{~L}$. Brillouin. Wave Propagation in Periodic Structures. Dover Publications, Inc., 1946. pp. 172-186.
21 Adrianus J. Dekker. Solid State Physics. Prentice-Hall, Inc., 1962. pp.238-250.

${ }^{22}$ Kenneth G. Lindh and Peter W. Likins. Infinite determinant methods for stability analysis of periodic-conflict differential equations. AIAA Journal, 8 No. 4:680-686, April 1970.

${ }^{23}$ John Dugundji and John $H$. Wendell. Some analysis methods for rotating systems with periodic coefficients. AIAA JOURNAL, 21 :890-897, June June 1983.

${ }^{24} \mathrm{P}$. Friedmann and L. J. Silverthorn. Aeroelastic stability of periodic systems with application to rotor blade flutter. AIA A Journal, 12 No.11:1559-1565, November 1974.

${ }^{25}$ David A. Peters and Kurt H. Hohenemser. Application of the floquet transition matrix to problems of lifting rotor analysis. Presented at the 26th Annual National Forum of the American Helicopter Society Washington D. C., June 1970. A70-34726.

${ }^{26}$ Edward N. Lorenz. Barotropic instability of rossby wave motion. Journal of the Atmospheric Sciences, $29: 258-264$, March 1972.

27 J. S. A. Green. Two-dimensional turbulence near the viscous limit. J. Fluid Mech, 62 :273-287, 1974.

${ }^{28}$ Kanefusa Gotoh, Michio Yamada, and Jiro Mizushima. The theory of stability of spatially periodic parallel flows. J. Fluid Mech., 127:45-58, 1983.

${ }^{29}$ Michio Yamada. Nonlinear stability theory of spatially periodic parallel flows. Journal of the Physical Society of Japan, 55No.9:3073-3079, September 1986.

${ }^{30} \mathrm{~K}$. Gotoh and M. Yamada. Stability of spatially periodic flows. In unknown, editor, Encyclopedia of Fluid Mechanics, chapter 19, pages 589-610, Houston, Tex: Gulf, 1986.

${ }^{31}$ M. G. Krein. Foundations of the theory of $\lambda$-zones of stability of a canonical system of linear differential equations with periodic coefficients. Four papers on ordinary differential equations, American Mathematical Society translations; ser. 2, v. 120:1-70, 1983. Translation of In Memoriam: Alesandr Aleksandrovic Andronov, Izdat. Akad. Nauk SSSR, Moscow, 1955, pp. 413-498.

32 J.W.S. Rayleigh. The Theory of Sound. Dover, 1945. Volume 2, chapter 21.

${ }^{33}$ P. G. Drazin and W.H. Reid. Hydrodynamic Stability. Cambridge University Press, 1981. Instability of unsteady flows pp. 353-369.

${ }^{34} \mathrm{C}$. S. Hsu. On approximating a general linera periodic system. Journal of Mathematical Analysis and Applications, 45 :234-251, 1974.

${ }^{35}$ Katsuhiko Ogata. State Space Analysis of Control Systems. Prentice-Hall,Inc., 1967.

${ }^{36} \mathrm{M}$. J. Lyell. Fluid column stability in the presence of periodic accelerations. AIA A Joumal, 31 No. 8:1519-1521, Aug. 1993.

${ }^{37}$ S. C. Sinha and D.-H. Wu. An efficient computational scheme for the analysis of periodic systems. Journal of Sound and Vibration, 151 No. 1:91-117, 1991.

${ }^{38}$ Christain von Kerczek and Stephen H. Davis. Linear stability theory of oscillatory stokes layers. J. Fluid Mech., 62 No.4:753-773, 1974.

39 Christopher G. Langton. Studying artificial life with cellular automata. Physical, 22 D :120-149, 1986.

${ }^{40}$ Chris G. Langton. Computation at the edge of chaos: phase 
transitions and emergent computation. Phsica $D, 42: 12-$ $37,1990$.

${ }^{41}$ M. Mitchell Waldrop. Complexity: the emerging science at the sdge of order and chaos. Simon \& Schuster, 1992.

42 Michael Barnsley. FRACTALS EVERYWHERE. Academic Press, Inc., 1988.

43 Michael Shub. Mysteries of mathematics and computation. The Mathematical Intelligencer, 16 No. 1:10-15, Winter 1994. 
Table 1 Integration results for $(\sinh (\eta / \sinh (1)))^{18}$ velocity profile

\begin{tabular}{ccc}
\hline \hline$\delta_{r}$ & $\Gamma$ & $c_{r}$ \\
\hline e $1.5430059290031 E-2$ & 0.2487720990379 & 0.9002607287015 \\
\hline \hline
\end{tabular}

Table 2 Integration results for $\eta^{6}$ velocity profile

\begin{tabular}{ccc}
\hline \hline$\delta_{r}$ & $\Gamma$ & $c_{r}$ \\
\hline-1.795749441083, & $7.2555284510676 E-2$ & $2.684018292084 E-2$ \\
$c_{i}$ & $=0.5, k=1.195$ &
\end{tabular}

Table 3 Integration results for Gaussian velocity profile

\begin{tabular}{ccc}
\hline \hline$\delta_{r}$ & $\Gamma$ & $c_{r}$ \\
\hline-1.76193559911 & $7.8446312448458 E-2$ & 0.1914746533746 \\
\hline \hline $0.4, k=0.9$ & &
\end{tabular}

Table 4 Exact Matrix Exponential Step Function Approximation Results for $(\sinh (\eta / \sinh (1)))^{18}$ velocity profile

\begin{tabular}{clllr}
\hline \hline Intervals, $K$ & $\delta_{r}$ & $\Gamma$ & $c_{r}$ & Time \\
\hline 12000 & 0.1324704157898 & 0.2394506125696 & 0.8954450299652 & 6106 \\
13000 & 0.1233419809349 & 0.2401785210625 & 0.8958327744015 & 6617 \\
14000 & 0.1155161824543 & 0.2408023920132 & 0.8961633816575 & 7127 \\
15000 & 0.108736216033 & 0.2413427747509 & 0.8964479072959 & 7639 \\
16000 & 0.1028055481471 & 0.2418153838495 & 0.8966953578756 & 8149 \\
17000 & $9.7573988718969 E-2$ & 0.24223222159 & 0.8969125382767 & 8658 \\
18000 & $9.2924800217857 E-2$ & 0.2426026126168 & 0.8971046810721 & 9157 \\
19000 & $8.8765848422101 E-2$ & 0.2429339134897 & 0.8972758834688 & 9677 \\
20000 & $8.5023484946906 E-2$ & 0.2432320028322 & 0.8974293902613 & 10188 \\
30000 & $6.1336692398649 E-2$ & 0.2451182120209 & 0.898389137051 & 15271 \\
35000 & $5.457366358024 E-2$ & 0.2456566231031 & 0.8986594651123 & 17813 \\
40000 & $4.9502716977565 E-2$ & 0.2460602929786 & 0.8988610967745 & 20377 \\
\hline
\end{tabular}

$c_{i}=0.4, k=0.115$ 
Table 5a Exact Matrix Exponential Step Function Approximation Results for $\eta^{6}$ velocity profile using exact $f^{\prime \prime}$

\begin{tabular}{clll}
\hline \hline Intervals,$K$ & $\delta_{r}$ & $\Gamma$ & $c_{r}$ \\
\hline 5000 & -1.731639624708 & $8.3398757988445 E-2$ & $2.663504066365 E-2$ \\
10000 & -1.763593751114 & $7.8166992537224 E-2$ & $2.6737302451565 E-2$ \\
20000 & -1.779569996798 & $7.5427110978254 E-2$ & $2.6788433789913 E-2$ \\
21000 & -1.780330756172 & $7.5294351899531 E-2$ & $2.6790868618323 E-2$ \\
\hline
\end{tabular}

$c_{i}=0.5, k=1.195$

Table 5b Exact Matrix Exponential Step Function Approximation

Results for $\eta^{6}$ velocity profile using numerical evaluation of $f^{\prime \prime}$

\begin{tabular}{clll}
\hline \hline Intervals, $K$ & $\delta_{r}$ & $\Gamma$ & $c_{r}$ \\
\hline 1000 & -1.476622305991 & 0.1178118119098 & $2.5823569373648 E-2$ \\
10000 & -1.764242903764 & $7.8057391206773 E-2$ & $2.6743768291609 E-2$ \\
20000 & -1.78021716754 & $7.5314187830809 E-2$ & $2.6794895155659 E-2$ \\
30000 & -1.785541787533 & $7.4379094561713 E-2$ & $2.6811937462023 E-2$ \\
\hline \hline
\end{tabular}

$c_{i}=0.5, k=1.195$

Table 6a Exact Matrix Exponential Step Function Approximation

Results for a Gaussian velocity profile using exact value of $f^{\prime \prime}$

\begin{tabular}{clll}
\hline \hline Intervals, $K$ & $\delta_{r}$ & $\bar{\Gamma}$ & $c_{r}$ \\
5000 & -1.743858151466 & $8.1434477636742 E-2$ & 0.1914012580744 \\
10000 & -1.752887165675 & $7.9954766508094 E-2$ & 0.1914378621255 \\
20000 & -1.757396473618 & $7.9206314493282 E-2$ & 0.1914561345435 \\
25000 & -1.758297914811 & $7.90559160312 E-2$ & 0.1914597866106 \\
30000 & -1.758898796215 & $7.8955517837813 E-2$ & 0.1914622208468 \\
\hline
\end{tabular}

$c_{i}=0.4, k=0.9$

Table 6b Exact Matrix Exponential Step Function Approximation Results for a Gaussian velocity profile using numerical evaluation of $f^{\prime \prime}$

\begin{tabular}{clll}
\hline \hline Intervals, $K$ & $\delta_{r}$ & $\Gamma$ & $c_{r}$ \\
\hline 1000 & -1.671129084883 & $9.2570372553067 E-2$ & 0.1911055992358 \\
5000 & -1.743886073843 & $8.1429939437306 E-2$ & 0.1914016096238 \\
10000 & -1.752915022464 & $7.9950162541102 E-2$ & 0.1914382134012 \\
20000 & -1.757424297442 & $7.9201676217921 E-2$ & 0.1914564856811 \\
30000 & -1.758926608932 & $7.8950867920244 E-2$ & 0.1914625719339 \\
\hline \hline
\end{tabular}

$c_{i}=0.4, k=0.9$ 

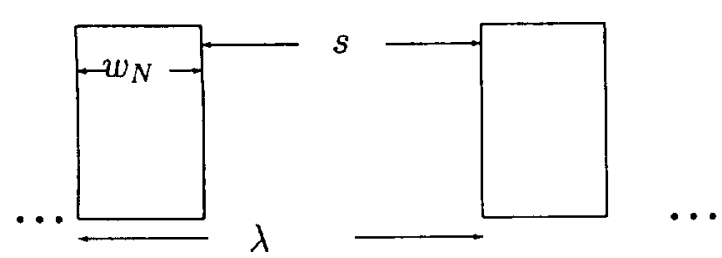

Fig. 1 Nozzle configuration.

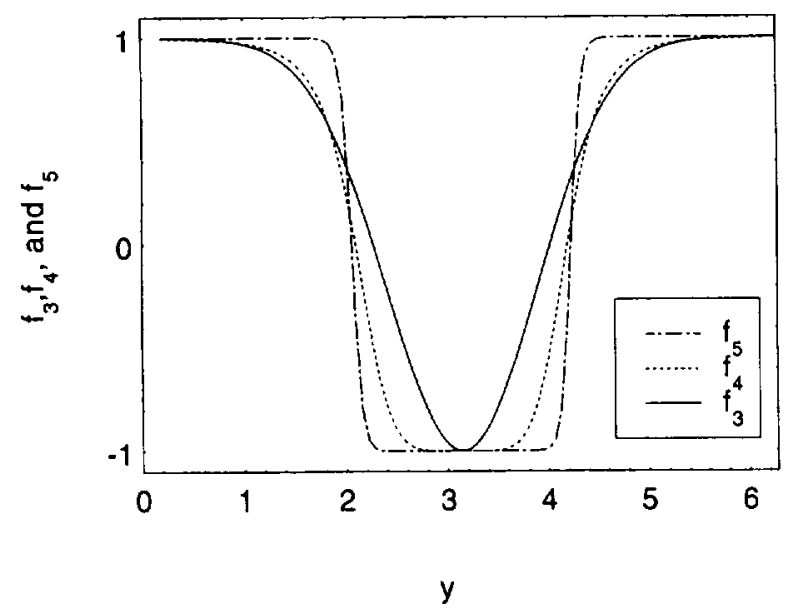

a) Velocity profiles.
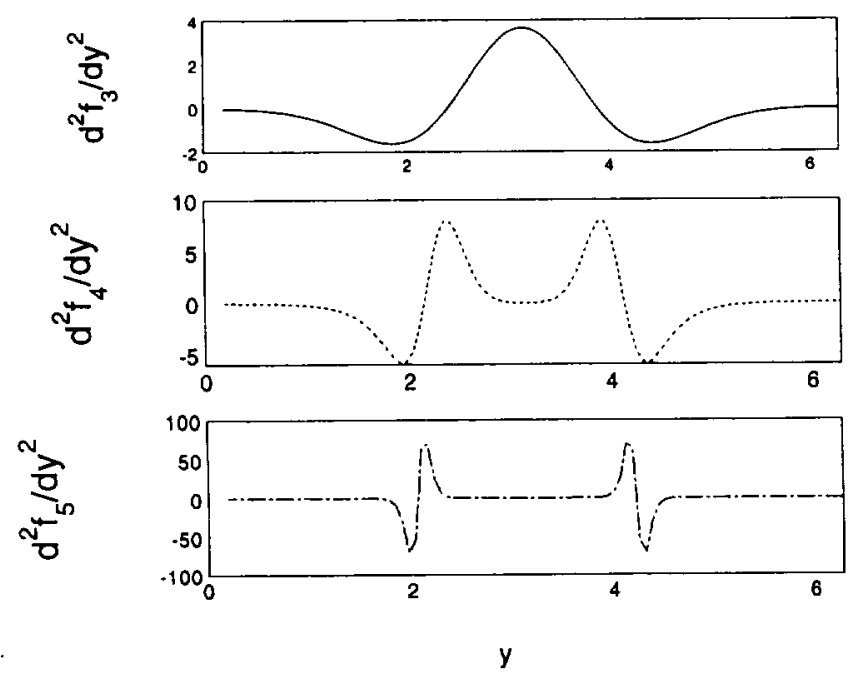

b) Second derivative of velocity profiles.

Fig. 2 Velocity profiles and second derivative of velocity profiles.

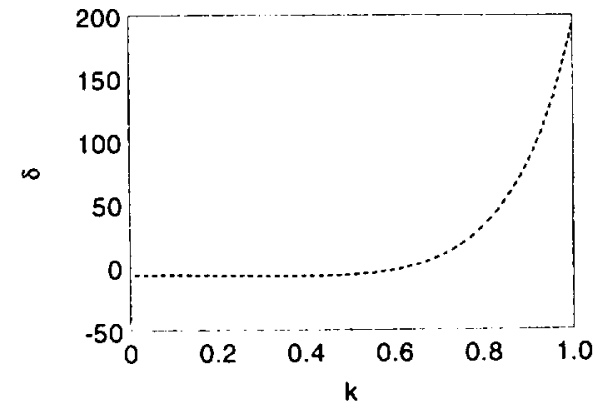

a) $\delta$ versus $k$ with $c=0$.

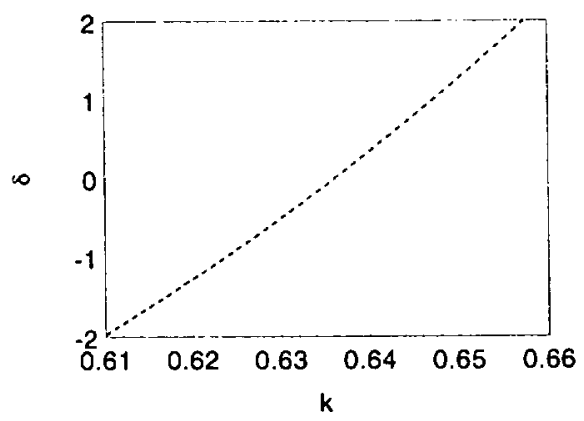

b) $\delta$ versus $k$ with $c=0$.

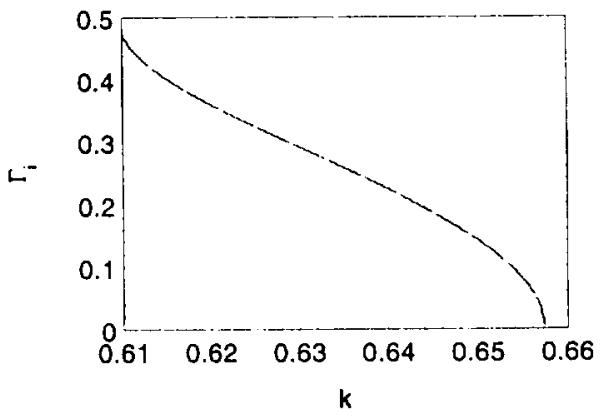

Fig. 3 Stability curves for broken line $v$ shaped velocity profile.

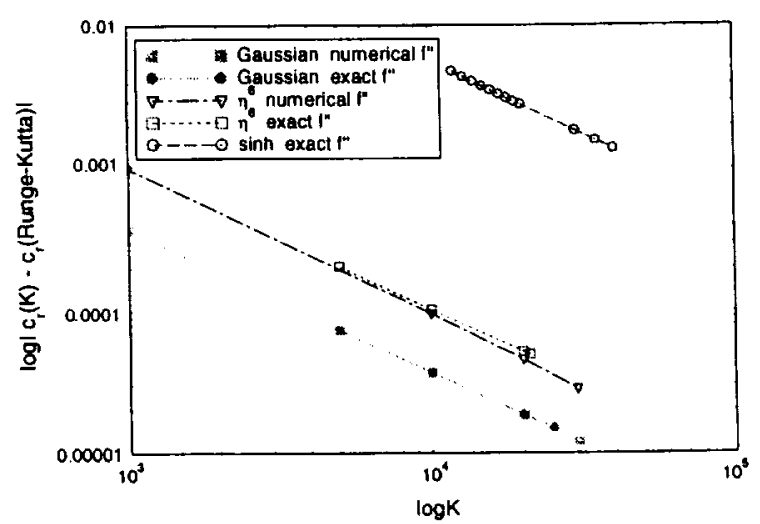

Fig. 4 Error in $c_{r}$ as a function of the number of intervals. 


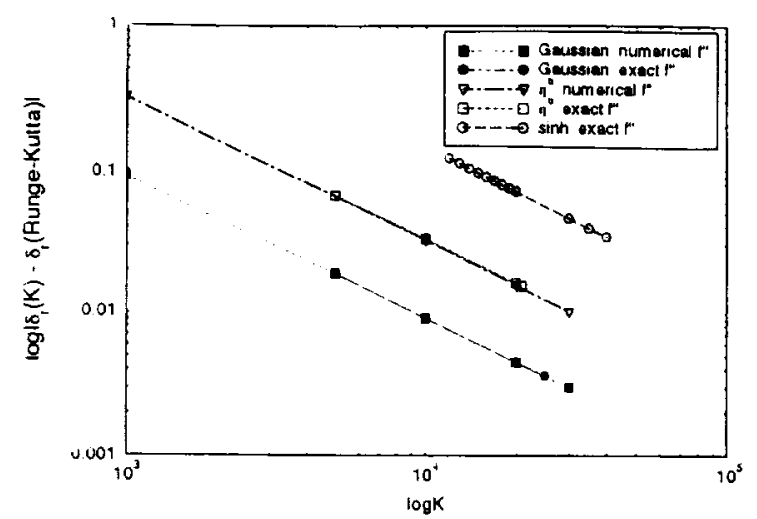

Fig. 5 Error in $\delta_{r}$ as a function of the number of intervals.

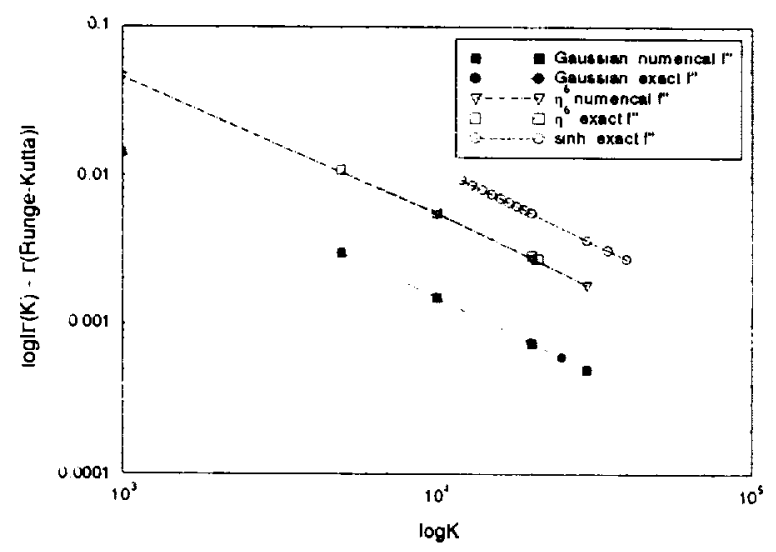

Fig. 6 Error in $\Gamma_{i}$ as a tunction of the number of intervals.

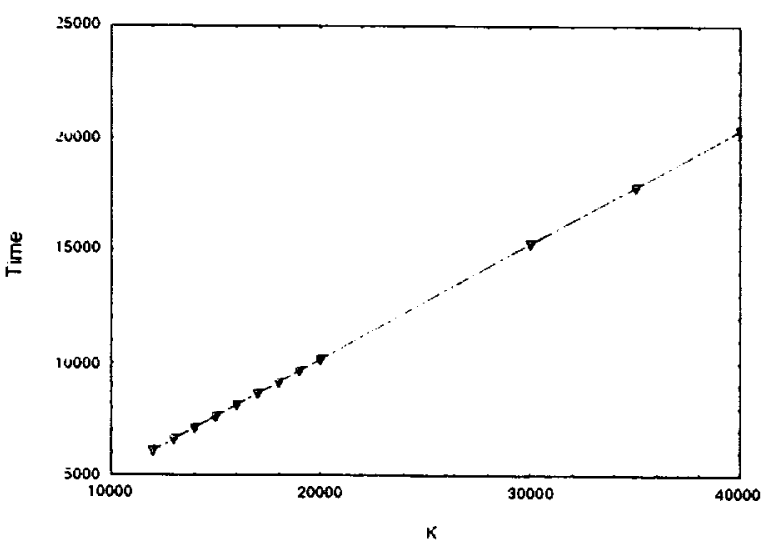

Fig. 7 Calculation time in seconds as a function of the number of intervals.

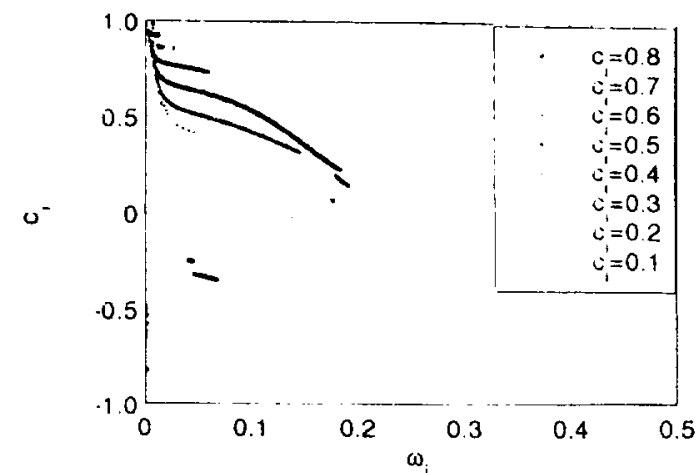

a) Eigenvalue $c_{r}$ versus growth rate ${ }^{\prime}{ }_{i}=\frac{k c_{i}}{2}$.

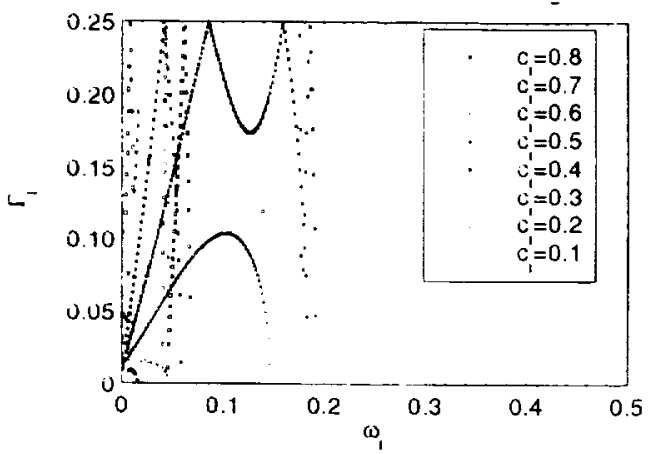

b) $\Gamma_{i}$ versus growth rate $\psi_{i}=\frac{k c_{i}}{2}$.

Fig. 8 Stability plots for $f_{3}$ velocity profile with $\Lambda=3$.

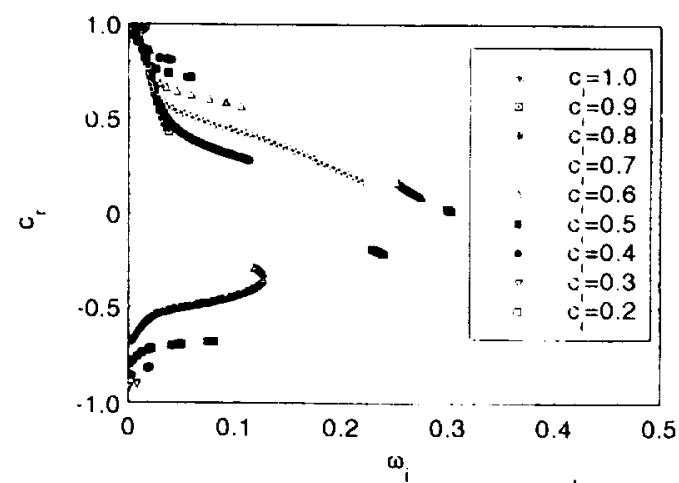

a) Eigenvalue $c_{r}$ versus growth rate $w_{i}=\frac{k c_{i}}{2}$

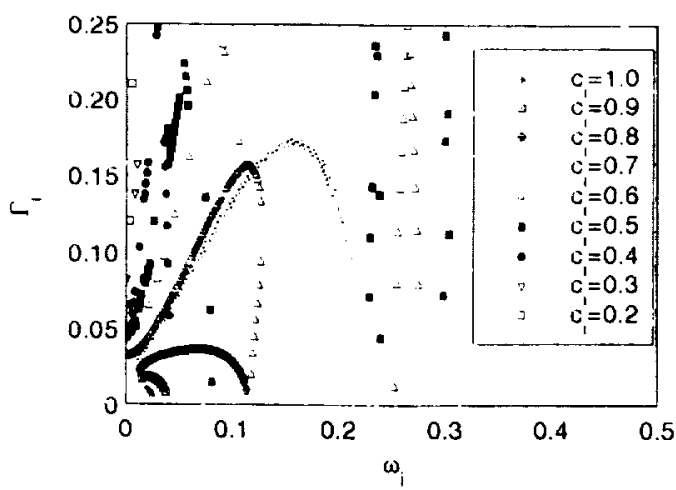

b) $\mathrm{l}_{i}$ versus growth rate $w_{i}=\frac{k c_{i}}{2}$.

Fig. 9 Stability plots for $f_{4}$ velocity profile with $\Lambda=3$. 


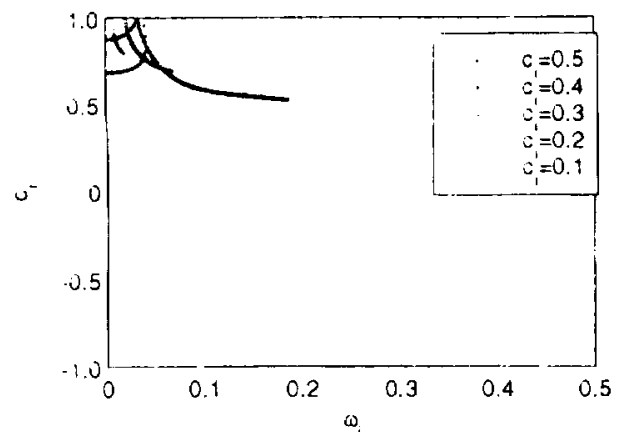

a) Eigenvalue $c$, versus growth rate $w_{i}=\frac{k_{i}}{2}$

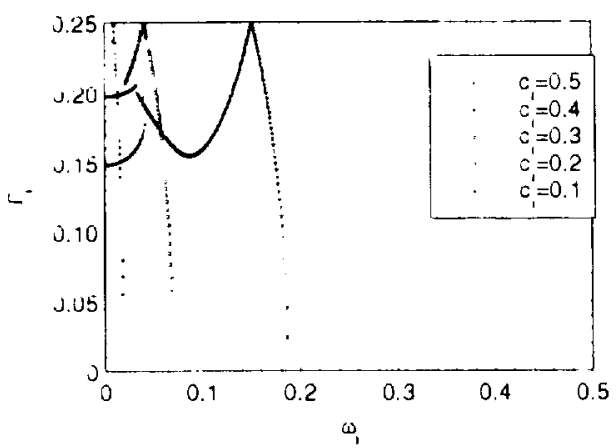

b) $\Gamma_{i}$ versus growth rate $w_{i}=\frac{k c_{i}}{2}$.

Fig. 10 Stability plots for $f_{5}^{\prime}$ velocity profile with $\Lambda=1.18$.

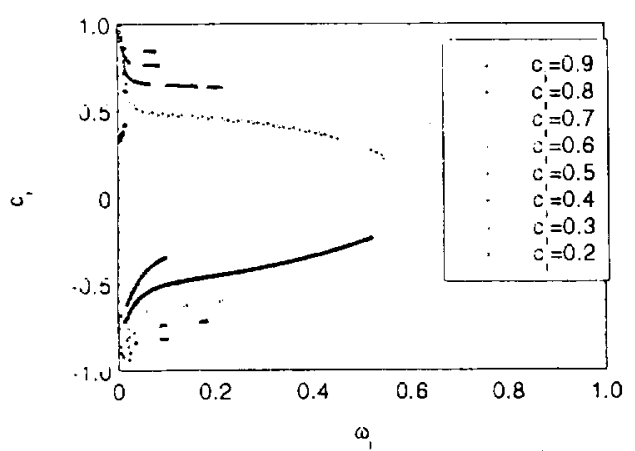

4) Eigenvalue $c_{r}$ versus growth rate $w_{i}=\frac{k c_{i}}{2}$.

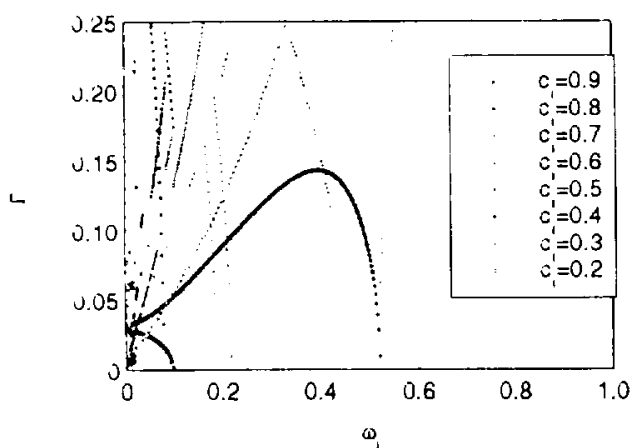

b) $\Gamma_{i}$ versus growth rate $w_{i}=\frac{k c_{i}}{2}$

Fig. 11 Stability plots for $f_{5}$ velocity profile with $\Lambda=1.5$

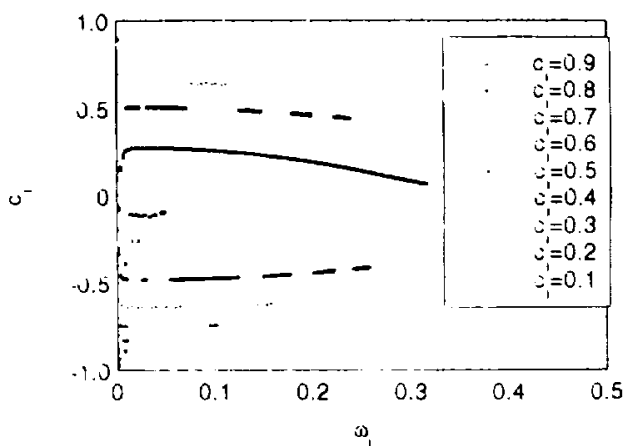

d) Eigenvalue $c_{j}$ lersus grow th rate $w_{i}=\frac{k c_{i}}{2}$

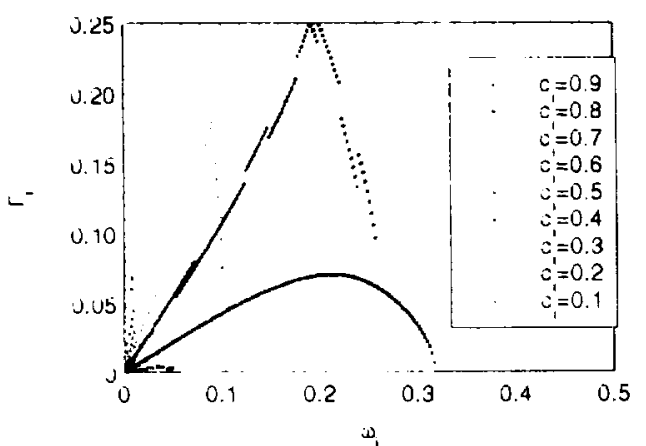

b) $\Gamma_{i}$ versus growth rate $w_{i}=\frac{k c_{i}}{2}$.

Fig. 12 Stability plots for $f_{5}$ velocity protile with $\Lambda=2.3$.

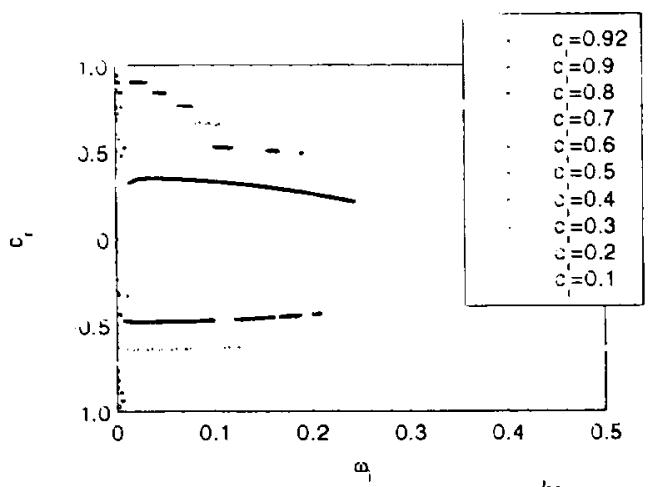

a) Eigenvalue $c_{r}$ versus growth rate $w_{i}=\frac{k_{i}}{2}$

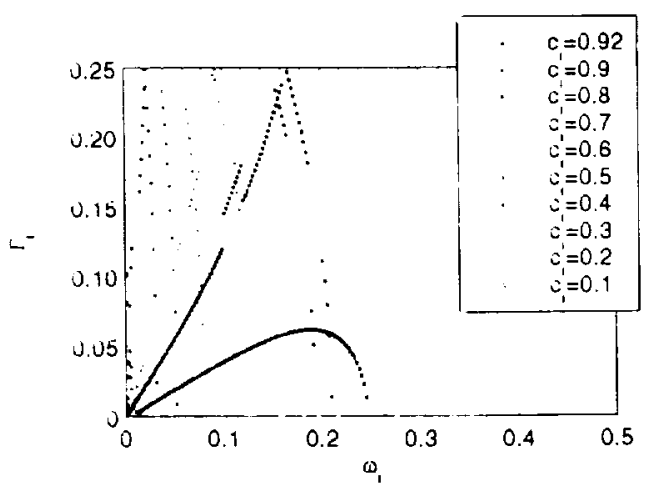

b) $\Gamma_{i}$ versus growth rate $w_{i}=\frac{k c_{i}}{2}$.

Fig. I3 Stability plots for $f_{5}$ velocity profile with $\Lambda=3$. 


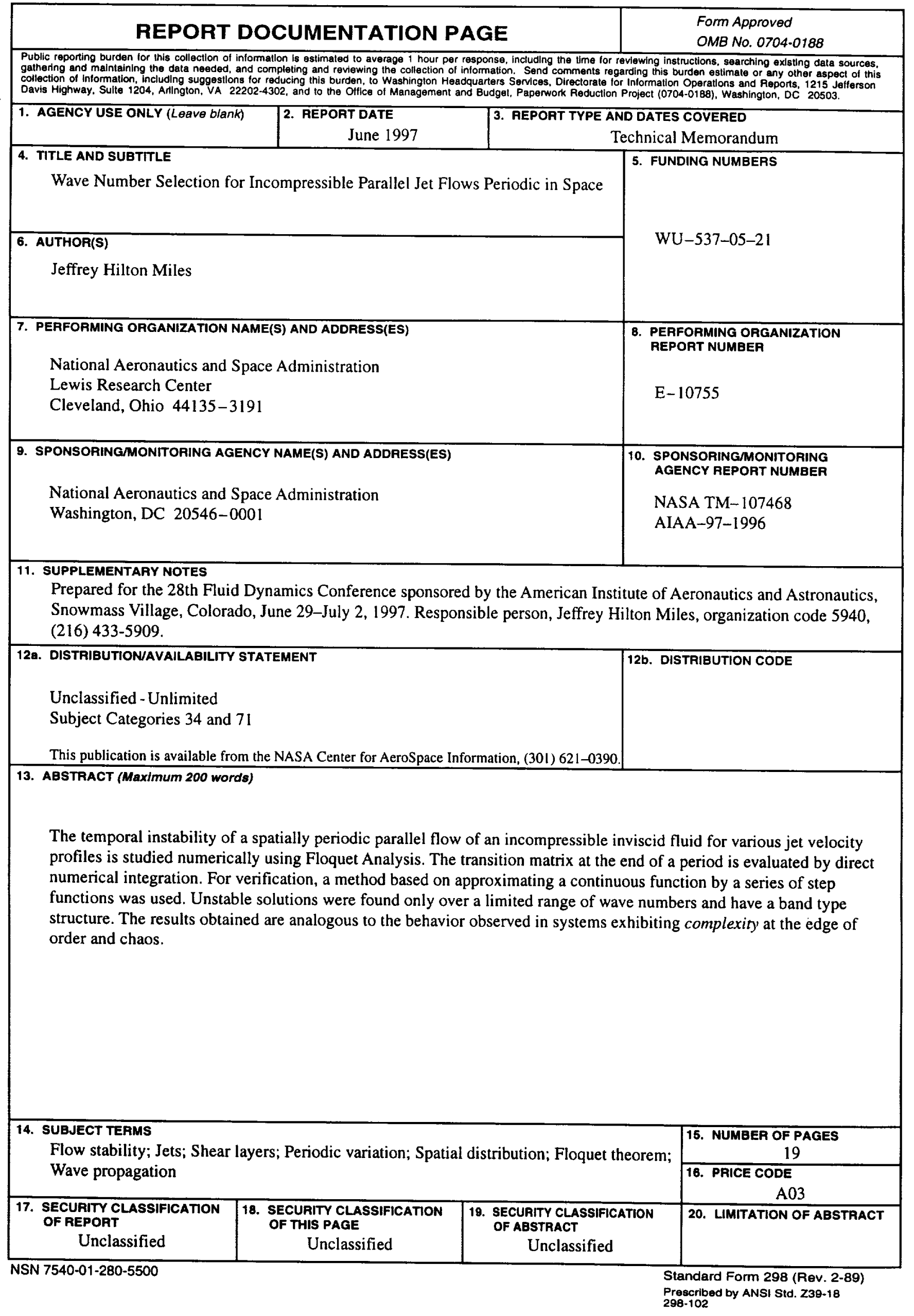

